\title{
Metastatic potential of human renal cell carcinoma: experimental model using subrenal capsule implantation in athymic nude mice
}

\author{
F. S. Grossi ${ }^{1,3}$, X. Zhao ${ }^{1}$, J. C. Romijn ${ }^{1}$, F. J. W. ten Kate ${ }^{2}$, and F. H. Schröder ${ }^{1}$ \\ Departments of ${ }^{1}$ Urology and ${ }^{2}$ Pathology, Erasmus University, Rotterdam, The Netherlands \\ ${ }^{3}$ Surgical Nephrology, University of Bari, Bari, Italy
}

Accepted: February 24, 1992

\begin{abstract}
Summary. The aim of this study was to determine whether subrenal capsule (SRC) implantation is a suitable model for the study of the metastatic potential of our human renal cell carcinoma (HRCC) lines and to establish new sublines with enhanced metastatic ability. NMRI athymic nude mice 7-11 weeks old received SRC implantation of our HRCC lines RC43 and RC21. These lines were not metastatic when implanted s.c. Mice were killed after 4 or 8 weeks, or when moribund. With the RC43 cell line the success rate for implantation was $69 \%$, with $89 \%$ of these showing metastases. The average volume of the implanted tumour fragments was $0.5 \mathrm{~mm}^{3}$ (range $0.28-0.7$ ), the average volume at the primary site at the time of death was $9087(9-32000) \mathrm{mm}^{3}$. Metastases were found in lymph nodes, liver, spleen, peritoneum, psoas muscle, pancreas, diaphragm and skin. The average volume of the metastases was $4139(0.5-9000) \mathrm{mm}^{3}$. Growing cell lines were established in vivo and in vitro from one splenic, one peritoneal, one diaphragmatic, and one hepatic metastasis. These sublines have faster in vivo and slower in vitro growth rates than the parental lines. With the RC21 cell line the success rate for implantation was $56 \%$ and the metastatic rate $78 \%$. The average volume of the implanted tumour was $0.8 \mathrm{~mm}^{3}(0.28-1.2)$, the average volume at the primary site at the time of death was $2685 \mathrm{~mm}^{3}(1.4-6534)$ and the average volume of metastases was $7.1 \mathrm{~mm}^{3}(0.5-37.5)$. Metastases were found in lymph nodes, lung and skin. No establishment was attempted for RC21 because of the small dimensions of these metastases. SRC implantation is thus considered a useful tool for the study of the metastatic ability of our cell lines RC43 and RC21. The establishment of new sublines with a faster growth rate and an enhanced metastatic ability will be useful for further studies on the metastatic process.
\end{abstract}

Key words: Metastatic potential - Subrenal capsule implantation - Human Renal Cell Carcinoma
Cancer patients rarely die of their primary tumours. Establishment of metastases in various organs and their subsequent growth usually cause the terminal cachexia of neoplastic patients.

The non-random pattern of visceral metastases suggested to Paget [1] more than 100 years ago that the growth of metastases was due to an affinity between tumour cells (seeds) and a suitable environment in some organs (soil). Since then, the metastatic process has only been partially clarified, mainly because of the relative scarcity of experimental models that could reliably reproduce the interactions between host and tumour cells.

Human renal cell carcinoma (HRCC) is an uncommon malignancy ( $6 \%$ of all tumours diagnosed each year) with a poor prognosis ranging from 73 to $8 \%$ survival at 5 years depending on the stage at diagnosis [2]. The establishment of HRCC lines growing in vitro or in vivo is not easy. In our laboratory only $10 \%$ of implanted tumours produced a serially transplantable line [3]. Furthermore, the simple model of an s.c.-growing tumour is not very useful for research regarding the metastatic process. None of our cell lines gave rise to metastases when implanted s.c. in nude mice, whereas all the patients from whom the tumours were isolated have since died.

The aim of this study was to determine whether orthotopic implantation under the renal capsule is a suitable method for the enhancement of the metastatic potential of our in vivo cell lines and to establish new sublines with enhanced metastatic ability.

\section{Materials and methods}

\section{Cell lines}

The two cell lines used, RC43 and RC21, are maintained in our laboratory. RC43 is a grade 4 renal cell carcinoma that was isolated following s.c. implantation in nude mice of a surgical specimen from a lymph node metastasis. The female patient with a complete staging (UICC 1978) pT3N4M0, died 3 month postoperatively because of cancer. The doubling time of this cell line is $84 \mathrm{~h}$ in vitro and 4 days in vivo. 
Table 1. Comparison of the results of the subrenal capsule (SRC) implantation for RC43 and RC21 cell lines

\begin{tabular}{lcc}
\hline & RC21 & RC43 \\
\hline Mean tumour size at implant $\left(\mathrm{mm}^{3}\right)$ & 0.8 & 0.5 \\
Success rate $(\%)$ & 55 & 69 \\
Metastatic rate $(\%)$ & 80 & 89 \\
Size of metastases $\left(\mathrm{mm}^{3}\right)$ & 7.2 & 4139 \\
Range & $0.5-37.5$ & $0.5-9000$ \\
Site of metastases & & \\
Lymph nodes & 10 & 10 \\
Lung & 5 & - \\
Liver & - & 4 \\
Spleen & - & 2 \\
Peritoneum & - & 2 \\
Skin & 1 & 1 \\
Diaphragm & - & 1 \\
Psoas muscle & - & 1 \\
Pancreas & - & 22 \\
Total number of sites & 16 & \\
\hline
\end{tabular}

$\mathrm{RC} 21$ is a grade 3 renal cell carcinoma that was isolated by implanting a specimen of a primary tumour from a female patient with complete staging pT2N0M1, who died of cancer 11 months postoperatively. The doubling time is $55 \mathrm{~h}$ in vitro and 5.2 days in vivo. Those two lines never resulted in metastases when implanted s.c.

\section{Animals}

NMRI nude mice from our breeding colony were used at 7-11 weeks old. The age of the mice was chosen to avoid the effects of low activity of natural killer cells, which is known to influence the expression of metastatic potential [4]. Mice were kept in a laminar flow cabinet throughout the experiments under specific pathogenfree conditions with free access to food and water.

\section{SRC implant}

The technique described by Bogden et al. [5] was used. Tumours were mechanically minced under a stereoscopic microscope in order to obtain fragments with average volume of approximately $0.5 \mathrm{~mm}^{3}$ ( $L \times W^{2} / 2 ; L=$ length, $W=$ width) as measured with a calibrated ocular micrometer.

The use of this technique minimizes the spilling of tumour cells from the implant site, which is possible when using a cell suspension for the implant. Mice, anaesthetized with $0.4 \mathrm{ml}$, tribromoethanol (Aldrich, Beerse, Belgium) i.p. were placed in the left lateral decubitus and the left kidney was exposed through a vertical flank incision. After a horizontal incision in the translucent kidney capsule, the tumour fragment was introduced beneath the renal capsule, using a 16-gauge trocar needle. The wound was closed in one layer with a $5 / 0$ silk suture.

Mice were killed at 4 and 8 weeks from the time of implantation, or when moribund. At autopsy the size of the tumour at the primary site was measured together with the size and location of macroscopically evident metastases. These were, when possible, removed in a sterile way to facilitate further growth in vitro and/or in vivo. Thoracic and abdominal organs were fixed in $10 \%$ formalin and processed for histological examination.
The growth rate in vivo was determined by caliper measurements of $L$ and $W$ of the SC implant every 3 days, using the formula for volume, $V,[6]$ :

$$
V=0.52(L \times W)^{3 / 2}
$$

The growth rate in vitro was determined by means of the MTT assay [7]. Differences were analysed for statistical significance by use of the $\chi$ test.

\section{Results}

RC43 cell line

Growth was seen in 9 out of 13 evaluable nude mice (Success rate $69 \%$ ); of these, $89 \%$ showed metastases (metastatic rate). The mean volume of the tumour at the implantation site was $9087 \mathrm{~mm}^{3}$ (range 9-32000). The average volume of metastases was $4139 \mathrm{~mm}^{3}(0.5-9000)$. Metastases were located (Table 1) in the lymph nodes (10), liver (4), spleen (2), peritoneum (2), contralateral psoas muscle (1), pancreas (1), skin (1) and diaphragm (1). No metastases were found in the lungs. No mice survived up to week 8 after implantation, all becoming cachectic within week 7 .

\section{RC21 cell line}

The success rate for implantation was 55\% (10 out of 18 evaluable). Metastatic rate was $80 \%$. The average volume at the primary site was $2685 \mathrm{~mm}^{3}(1.4-6534)$ and the average volume at the metastatic location was $7.2 \mathrm{~mm}^{3}$ (0.5-37.5). Metastases were situated in the lymph nodes (10), lung (5) and skin (1). Only $30 \%$ of mice survived 8 weeks from implantation.

\section{Establishment of new sublines}

Four new sublines were established, derived from RC43 metastases. No sublines were isolated from RC21 because of the small dimensions of the metastases.

The RC43MSP subline was isolated from a splenic metastasis. It is maintained in vitro, with a doubling time of $100 \mathrm{~h}$ and in vivo, by SC implant, with a doubling time of 3 days. The RC43MLI subline was isolated from a liver metastasis and has a doubling time of $105 \mathrm{~h}$ in vitro and 2.5 days in vivo. RC43MDI was isolated from a diaphragmatic metastasis with a doubling time of $95 \mathrm{~h}$ in vitro and 2.5 days in vivo. RC43MBO was isolated from a colon metastasis and shows a doubling time of $100 \mathrm{~h}$ in vitro and 3 days in vivo.

As shown in Fig. 1, all these sublines exhibited a faster growth rate in vivo than the parental $\mathrm{RC} 43$, while they have a slower growth rate in vitro $(P$ of RC43 vs MSP $=$ 0.002 , vs $\mathrm{MBO}=0.004$, vs $\mathrm{MLI}=<0.01$, vs $\mathrm{MDI}=$ 0.001 ).

No histological changes were evident; these sublines remained G4 renal cell carcinoma as the parental RC43. When reimplanted in the SRC, all sublines spread fast, 


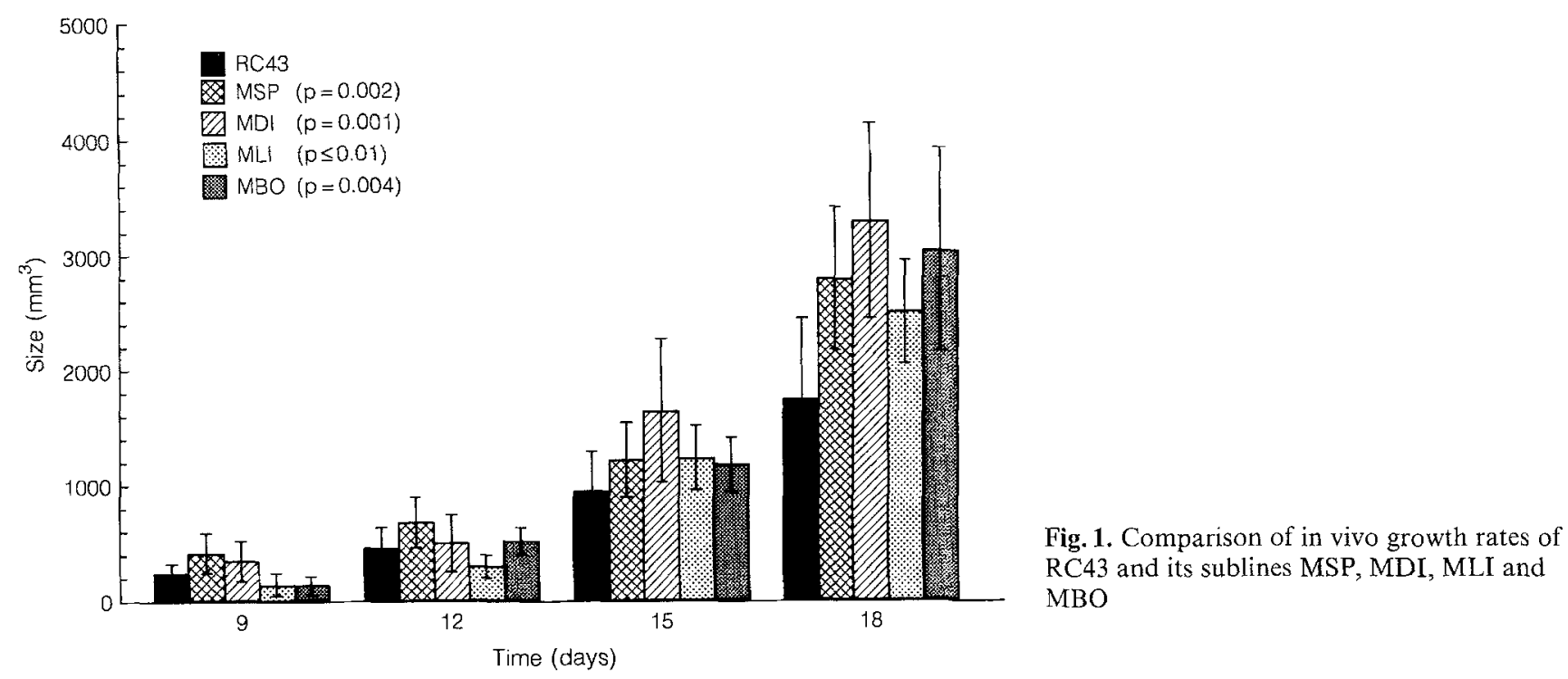

Table 2. Comparison of the results of (SRC) implantation for the sublines MBO, MSP, MDI, MLI

\begin{tabular}{lcccc}
\hline & MSP & MBO & MDI & MLI \\
\hline Mean tumour size at implant $\left(\mathrm{mm}^{3}\right)$ & 0.6 & 1.2 & 1 & 1.1 \\
Success rate $(\%)$ & 100 & 100 & 100 & 100 \\
Metastatic rate $(\%)$ & 100 & 100 & 100 & 100 \\
Total number of metastases & $99^{*}$ & $97^{*}$ & $98^{*}$ & $149^{*}$ \\
\hline
\end{tabular}

* $P$-value for difference from $\mathrm{RC} 43$ parental line $=0.001$

which further reduced the survival time of the mice (Table 2). Site specificity of these sublines with respect to metastases is under investigation.

\section{Discussion}

As previously stated, metastases are the most probable cause of death in cancer patients. The metastatic process has been only partially clarified. It is now known to be a multistep process involving neovascularization, invasion of the venules, capillaries and lymphatics, embolization, arrest in a distant capillary district, adherence to the endothelial cells, extravasation and, finally, tumour cell proliferation [8].

The study of these different steps requires experimental models that reproduce physiological conditions occuring in the human. After SC tumour implantation, a fibrous capsule surrounding the tumour is seen as part of the reaction of the host animal [3], while local immune factors also play a role in initiating or inhibiting the metastatic cascade. The SRC assay was first described by Bogden [5, $9,10]$, who used this method for testing the sensitivity of tumours of various origins to different chemotherapeutic agents. Following SRC implantation no fibrous capsule is formed by the animal [3] and the vascularization at the site of the implant seems to aid full expression of the metastatic ability of the cells [8].

Recently the concept that orthotopic implantation is essential for selection, growth and formation of metastases was suggested by Fidler and Naito [11] for HRCC and by other authors for different tumour systems [12177.

From analysis of our results, no statistically significant difference was found between the absolute number of colonies in the two different cell lines $(P=0.25)$. In contrast, the difference in size of the tumour at the implantation site at the time of death $(P=0.005)$ and the difference in size of the metastatic lesions $(P=0.001)$ are highly significant and favour the RC43-derived metastases.

This difference can only be partially ascribed to the faster growth rate of $\mathrm{RC} 43$ compared to $\mathrm{RC} 21$. Whereas the average tumour volume at the primary site is only 3 times larger for RC43, a 100-fold difference was observed for the average volume of metastases. Observing the localization of metastases, it is interesting to note that while RC21 favours lung colonization, RC43 shows wide colonization of abdominal organs and of the liver. This difference could be due to a different route of metastases: haematogenous for RC21 and via the lymphatics for $\mathrm{RC} 43$, particularly as RC43 was originally isolated from a lymph node metastasis.

The sublines that we have isolated have some interesting characteristics. By flow cytometry (data not shown) a difference in the ploidy pattern between at least two of the sublines (MSP and MDI) and the parental RC43 was observed. The observation that growth was faster in vivo and slower in vitro for all sublines with respect to the parental RC43 cell line also strongly supports in their origin from different clones of the four sublines.

We conclude that SRC implantation is a useful tool for the evaluation of the metastatic potential of our in vivo cell lines, RC43 nd RC21. With the SRC implantation it is also possible to establish new sublines, allowing expression of the intrinsic clonal heterogeneity of HRCC. 
The enhanced metastatic ability can be used for further research on metastatic processes.

\section{References}

1. Paget S (1989) The distribution of secondary growth in cancer of breast. Lancet $\mathrm{I}: 571$

2. Johnson DE, Swanson DA, Von Eschenbach A (1988) Tumors of the genitourinary tract. In: Tanagho EA, McAnich JW (eds) Smith's General Urology, 12th ed. Prentice Hall, Englewood Cliffs, NJ, p 310

3. Kurth KH, Romijn JC, Van Dongen JW, Lieber MM, Schroeder FH (1986) Experimental chemotherapy of renal adenocarcinoma. In: deKernion JB, Pavone-Macaluso $M$ (eds) Tumors of the kidney. Williams \& Wilkins, Baltimore, Md, p 228

4. Hanna H (1980) Expression of metastatic potential of tumor cells in young mice is correlated with low levels of NK cell-mediated cytotoxity. Int J Cancer 26:675

5. Bogden AE, Haskell PM, lePage DJ, Kelton DE, Cobb WR, Esber HJ (1979) Growth of human tumour xenografts implanted under the renal capsule of normal immunocompetent mice. Exp Cell Biol 47:281

6. Van Steenbrugge GJ, Groen M, Romijn JC, Schroeder FH (1984) Biological effects of hormonal treatment regimens on a transplantable human prostatic tumor line. J Urol 131:812

7. Romijn JC, Verkoelen CF, Schroeder FH (1988) Application of the MTT assay to human prostate cancer cell lines in vitro: establishment of test conditions and assessment of hormonestimulated growth and drug-induced cytostatic and cytotoxic effects. Prostate 12:99

8. Fidler IJ (1990) Critical factors in the biology of human cancer metastasis: Twenty-eight G.H.A. Clowes Memorial Award lecture. Cancer Res 50:6130

9. Bogden AE, Cobb WR, lePage DJ, Haskell PM, Gulkin TA, Ward A, Kelton DE, Esber HJ (1981) Chemotherapy respon- siveness of human tumors on first transplant generation xenografts in the normal immunocompetent mouse: 6 days subrenal capsule assay. Cancer 48:10

10. Bogden AE, Von Hoff DD (1984) Comparison of the human tumour cloning and subrenal capsule assay. Cancer Res 44:1087

11. Fidler IJ, Naito S, Pethak S (1990) Orthotopic implantation is essential for the selection, growth and metastasis of human renal cell carcinoma in nude mice. Cancer Metastasis Rev 9:149

12. Tabuchi Y, Nakamura T, Saitoh Y (1991) Liver metastases induced by implantation of VX2 cancer in to the gastrointestine. J Surg Res $50: 216$

13. Ibrahiem EHI, Nigam VN, Brailosky CA, Nadermas $P$, Elhilali M (1983) Orthotopic implantation of primary ( $N$-[4-(5-nitro-2furyl)-2-thiazolyl])formamide-induced bladder cancer in bladder submucosa: an animal model for bladder cancer study. Cancer Res 43:617

14. Morikawa K, Walker SM, Jessup JM, Fidler IJ (1988) In vivo selection of highly metastatic cells from surgical specimens of different human colon carcinoma implanted in to different organs of nude mice. Cancer Res 48:1943

15. Bresalier RS, Raper SE, Hujanen ES, Kim YS (1987) A new model 'for human colon cancer metastasis. Int J Cancer 39:625

16. Kerbel RS, Man MS, Dexter D (1984) A model of human cancer metastasis: extensive spontaneous nd artificial metastases of a human pigmented melanoma nd derived variant sublines in nude mice. J Natl Cancer Inst 72:92

17. Shafie LLA (1980) Formation of metastases by human breast carcinoma cells (MCF-7) in nude mice. Cancer Lett 11:81

Dr. Francesco Saverio Grossi

Cattedra di Nefrologia di Interesse Chirurgico

Università degli Studi di Bari

c/o Policlinico

Piazza Giulia Cesare, 11

I-70124 Bari

Italy 